\title{
Screening women for intimate partner violence in healthcare settings: abridged Cochrane systematic review and meta-analysis
}

\author{
OPEN ACCESS
}

\begin{abstract}
Lorna J O'Doherty honorary fellow ${ }^{1}$, Angela Taft professor ${ }^{2}$, Kelsey Hegarty professor ${ }^{1}$, Jean Ramsay honorary senior research fellow ${ }^{3}$, Leslie L Davidson professor ${ }^{4}$, Gene Feder professor ${ }^{5}$

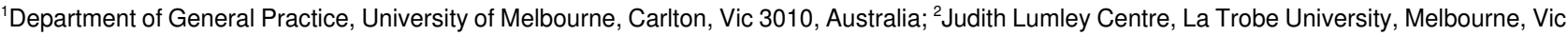
3000, Australia; ${ }^{3}$ Centre for Primary Health Care and Public Health, Barts and The London School of Medicine and Dentistry, Queen Mary College, University of London, London, UK; ${ }^{4}$ Department of Epidemiology, Mailman School of Public Health, Columbia University, New York, NY, USA; ${ }^{5}$ Centre for Academic Primary Care, School of Social and Community Medicine, University of Bristol, Bristol, UK
\end{abstract}

\begin{abstract}
Objective To examine the effectiveness of screening for intimate partner violence conducted within healthcare settings to determine whether or not screening increases identification and referral to support agencies, improves women's wellbeing, decreases further violence, or causes harm.

Design Systematic review and meta-analysis of trials assessing effectiveness of screening. Study assessment, data abstraction, and quality assessment were conducted independently by two of the authors. Standardised estimations of the risk ratios and $95 \%$ confidence intervals were calculated.
\end{abstract}

Data sources Nine databases searched up to July 2012 (CENTRAL, Medline, Medline(R), Embase, DARE, CINAHL, PsycINFO, Sociological Abstracts, and ASSIA), and five trials registers searched up to 2010.

Eligibility criteria for selecting studies Randomised or quasi-randomised trials of screening programmes for intimate partner violence involving all women aged $\geq 16$ attending a healthcare setting. We included only studies in which clinicians in the intervention arm personally conducted the screening, or were informed of the screening result at the time of the consultation, compared with usual care (or no screening). Studies of screening programmes that were followed by structured interventions such as advocacy or therapeutic intervention were excluded.

Results 11 eligible trials $(n=13027)$ were identified. In six pooled studies $(n=3564)$, screening increased the identification of intimate partner violence (risk ratio $2.33,95 \%$ confidence interval 1.39 to 3.89 ), particularly in antenatal settings $(4.26,1.76$ to 10.31$)$. Based on three studies $(n=1400)$, we detected no evidence that screening increases referrals to domestic violence support services $(2.67,0.99$ to 7.20$)$. Only two studies measured women's experience of violence after screening (three to 18 months after screening) and found no reduction in intimate partner violence. One study reported that screening does not cause harm.

Conclusions Though screening is likely to increase identification of intimate partner violence in healthcare settings, rates of identification from screening interventions were low relative to best estimates of prevalence of such violence. It is uncertain whether screening increases effective referral to supportive agencies. Screening does not seem to cause harm in the short term, but harm was measured in only one study. As the primary studies did not detect improved outcomes for women screened for intimate partner violence, there is insufficient evidence for screening in healthcare settings. Studies comparing screening versus case finding, or screening in combination with therapeutic intervention for women's long term wellbeing, are needed to inform the implementation of identification policies in healthcare settings.

\section{Introduction}

Intimate partner violence is a major cause of death and disability on a worldwide scale. ${ }^{12}$ The World Health Organization (WHO) highlights violence against women as a priority health issue, with one in three women globally experiencing physical and/or sexual violence from a partner. ${ }^{23}$ While the rates of intimate partner violence differ in low, middle, and high income regions, the health effects are similar across the globe. ${ }^{2}$ In all countries, women experiencing such violence often present in health settings and require wide ranging medical services. ${ }^{4}$ Since the late 1990s, many health professional associations have published clinician guidelines on how to identify and respond to women who have been abused, ${ }^{5}$ and health professionals are increasingly 
required to undertake screening in accordance with national health policies. ${ }^{67}$ In some countries, screening is advocated without sufficient resources or referral options in place, and clinicians often lack skills and knowledge to respond appropriately because of a lack of training. ${ }^{8}$ The WHO clinician guidelines recommend against screening, particularly in low to middle income health settings. ${ }^{5}$ On the other hand, a recent systematic review to update the 2004 recommendations from the US Preventive Services Task Force supported screening but included only a subset of trials relevant to the United States. ${ }^{9}$

Screening in healthcare settings aims to identify women with current or past experience of violence from an intimate partner or ex-partner so they can be referred to, or be offered, other (therapeutic) interventions leading to beneficial outcomes. There are, however, many barriers that influence the capacity of women to disclose such events and that impact on the effectiveness of screening. ${ }^{10}$ Intimate partner violence is therefore problematic when traditional screening criteria are applied ${ }^{11}$ because it is a complex social phenomenon rather than a disease. Nonetheless, the decision to implement screening programmes still requires evidence of effectiveness. It is important to distinguish between options of universal screening (application of a standardised question to all women without "symptoms" according to a procedure that varies little from place to place), selective screening (when high risk groups, such as pregnant women or those seeking terminations of pregnancy, are screened), routine inquiry (when all women are asked but the method/question might vary according to the provider or woman's situation - for example, taking a social history in a clinical setting), and case finding (asking if indicators of risk are present). ${ }^{12}$ For the purposes of this review, we included studies of universal and selective screening, with the added proviso that the clinician be aware of the woman's abuse status (either through direct enquiry with the women themselves or being notified of a positive result of screening). Studies of interventions that went beyond inquiry and initial support/referral were excluded given that it is not feasible for most health professionals to deliver more intensive therapies (because of lack of time and skill for advocacy). More importantly, however, it was necessary to isolate the screening effect from other follow-up interventions to provide evidence of the independent contribution of screening.

Based on a recently published Cochrane Review, ${ }^{12}$ we reviewed the evidence for health service screening. Our review question was whether screening women for intimate partner violence in healthcare settings is safe, effective, and cost effective. We hypothesised that screening can, as a first step, lead to improved identification, information giving, and referral to support services (primary outcomes), which in turn might lead to a reduction in abuse and an improvement in health and wellbeing (secondary outcomes) in the longer term. ${ }^{13}{ }^{14}$

\section{Methods}

The protocol for this review can be accessed through the Cochrane Library. ${ }^{15}$

\section{Inclusion criteria}

Types of studies-We included any studies that allocated individual women or clusters of women by a random or a quasi-random method (for example, alternate allocation) to a screening intervention compared with usual care (or in a trial with a condition when clinicians were not aware of women's screening results).
Participants - Studies included women (aged $\geq 16$ ) attending a healthcare setting, including general (family) practice; antenatal and postnatal services; hospital emergency, inpatient or outpatient services; specialists (such as obstetrics and gynaecology, psychiatry); community health services; drug and alcohol services; mental health services.

Interventions-Screening was defined as any of a range of methods (face to face, written, or computerised survey, involving specific inquiry about intimate partner violence or inquiry about intimate partner violence as part of general psychosocial screening) that aimed for all women patients in a healthcare setting to be asked about their experience of such violence. This included the use of validated screening tools as well as simply asking one or a range of questions related to intimate partner violence on one or several occasions. We included only studies in which clinicians in the intervention arm personally conducted the screening, or were informed of the screening result at the time of the consultation, and women in the control groups received usual care or, when screening was conducted for research purposes (such as to establish participant eligibility), the clinicians were not informed of (blinded to) the result of screening and therefore could not act on the information. We excluded studies of interventions that involved structured clinical intervention after screening.

\section{Search methods}

We ran three searches for this review (from their start dates to September 2009, updated in September 2011 and July 2012) using terms for intimate partner violence, identification, and referral appropriate to the following databases: Cochrane Central Register of Controlled Trials, Medline, Medline(R), Embase, DARE, CINAHL PLUS, PsycINFO, ASSIA, and Sociological Abstracts. We searched the following trial registers to 2010: $m e t a$ Register of Controlled Trials ( $m \mathrm{RCT}$ ), WHO International Clinical Trials Registry Platform, ClinicalTrials.gov, Australian New Zealand Clinical Trials Registry, and International Standard Randomised Controlled Trial Register. We also searched the websites of the WHO (www.who.int/topics/violence/en), Violence Against Women Online Resources (www.vaw.umn. edu), and Domestic Violence Data Source (www.lho.org.uk/ viewResource. aspx ?id=9443) and contacted the WHO Violence and Injury Programme to inquire about any screening studies fitting our criteria. We also examined the reference lists of included papers and performed forwards and backwards citation tracking.

\section{Data collection}

Two reviewers (LO'D and AT) independently assessed abstracts. If reviewers disagreed about abstract inclusion, this was resolved by reading the full study followed by discussion. A third reviewer $(\mathrm{KH})$ was consulted to resolve any remaining difference of opinion. A similar process was followed in selecting studies from the full text articles retrieved. Two reviewers (LO'D and AT) independently extracted data from papers included in the review and entered them onto an electronic data collection form. The primary outcomes comprised:

- Identification of intimate partner violence (data based on clinical encounter)

- Information giving and referrals to support agencies (including take-up rates when available).

The secondary outcomes comprised:

- Intimate partner violence 
- Women's physical and psychosocial health

- Occurrence of adverse outcomes

- Cost benefit.

Any missing information or clarification was requested from corresponding authors. Data were entered into Review Manager (Revman) 5.1 software. ${ }^{16}$ Two reviewers (LO'D and AT) assessed risk of bias using Revman's risk of bias assessment tool. ${ }^{17}$

\section{Data analysis}

For binary outcomes, we calculated a standard estimation of the risk ratio with random effects and a $95 \%$ confidence interval from studies that could be pooled. ${ }^{17}$ For outcomes for which data from studies could not be pooled (because of lack of comparable studies - for example, heterogeneous methods of measurement or insufficient data being reported or available from authors), we reported the results as reported in individual studies. Missing data and dropout rates were assessed by comparing numbers analysed with numbers enrolled and by examining the variation in denominators. We used published imputed figures for missing data (to report the results) when available. Participants were analysed in the groups to which they were randomised. We used random effects models to manage study heterogeneity ${ }^{16}$ and performed subgroup analyses for type of healthcare setting. We calculated absolute benefit by calculating assumed benefit (unscreened) by summing the number of identified or referred but unscreened women and then calculated this for the rate per 1000 . We then calculated the corresponding benefit (screened) and confidence intervals by multiplying the assumed benefit by the risk ratios and confidence intervals of unscreened to screened women. Finally, absolute benefit was calculated as $1000 \times \mathrm{ACR} \times(1-\mathrm{RR})$, where ACR is the assumed control risk based on the number per 1000 and RR is the risk ratio. ${ }^{17}$ To test for heterogeneity, we included the $\mathrm{I}^{2}$ statistic and $95 \%$ confidence interval to measure the proportion of variation of the estimated treatment effect attributable to heterogeneity across the studies, rather than to sampling error. The $\mathrm{I}^{2}$ statistic was interpreted according to Cochrane guidelines, with a result of $0-29 \%$ being low, 30-50\% moderate, and 50-90\% considerable heterogeneity, with a significance of $\mathrm{P}=0.05 .{ }^{17} \mathrm{We}$ used the GRADE evaluation of evidence to grade the review findings: high quality (further research is unlikely to change our confidence in the estimate of effect), moderate quality (further research is likely to have an important impact on our confidence in the estimate of effect and might change it), and low quality (further research is likely to have an important impact on our confidence in the estimate of effect and is likely to change it). ${ }^{18}$

\section{Results}

Searches generated 6513 unique abstracts (fig $1 \Downarrow$ ). We included 11 studies in the review (see appendix for full details of study characteristics); all were conducted in high income countries and the 13027 women recruited were sociodemographically diverse. Women recruited in antenatal clinics tended to be younger, while those in emergency clinics were older, but all were in their reproductive years. Three studies were evaluated as "high risk" on four of the seven criteria for risk of bias, ${ }^{19-21}$ with four studies scoring "low risk" on four criteria (table $1 \Downarrow$ ). ${ }^{22-25}$ Dropout rates were low (see appendix), with the exception of the study by MacMillan and colleagues ${ }^{26}$ (which had substantially longer follow-up periods). Though they reported imputed data, none of the data reported were meta-analysed in our review. The only other study to report imputed data was by Ahmad and colleagues ${ }^{25}$; we used their imputed figures for detection in our (identification) meta-analysis.

\section{Identification by clinician}

We pooled six studies in a meta-analysis of the effect of screening on identification of intimate partner violence (fig $2 \Downarrow$ ); all but one ${ }^{20}$ consisted of a computer assisted self completion screening process, with positive results conveyed to clinicians (see appendix). Each study measured "identification" in such a way that data were comparable (for instance, based on clinical encounter). Screening increased identification by $133 \%$ (risk ratio $2.33,95 \%$ confidence interval 1.39 to 3.89 ) compared with usual care. In most cases, the proportion of women identified was small, ranging from $3 \%{ }^{21}$ to $17 \% .^{25}$

Five studies were excluded from the meta-analysis of identification of exposure to intimate partner violence. Koziol-McLain and colleagues examined re-victimisation, ${ }^{23}$ and MacMillan and colleagues selected intimate partner violence and women's quality of life as primary outcomes ${ }^{26}$ For the latter study, of all women with positive results on screening, we estimated identification based on proportions of women who discussed intimate partner violence with their clinicians in the screened $(44 \%, 88 / 199)$ versus non-screened $(8 \%, 17 / 212)$ groups. We could not, however, include this study in the main analysis in which the denominator was all enrolled women. Kataoka and colleagues reported only prevalence rates in written $(29 \%, 48 / 163)$ versus face to face $(19 \%, 32 / 165)$ inquiry. ${ }^{22}$ Similarly, the 2006 study by MacMillan and colleagues reported on prevalence (4.1-17.7\% at 12 months) across emergency departments, family practices, and women's clinics. ${ }^{19}$ Klevens and colleagues compared disclosure to clinicians $(8.7 \%, 4 / 46)$ with women's disclosure via audio computer assisted self interviews (A-CASI) $(21.3 \%, 17 / 80) .{ }^{24}$ While this study did report that three women (of 80) in the A-CASI group subsequently discussed abuse with their clinician, identification was not measured consistently across the groups and therefore we excluded it.

\section{Information giving, referrals, and take up of services}

Three studies were included in the meta-analysis of referrals (fig $3 \Downarrow$ ). These were referrals to social workers, ${ }^{21}$ an in house advocacy programme and other off site services, ${ }^{24}$ and an on site nurse counsellor. ${ }^{25}$ While the pooled effect (risk ratio 2.67, $95 \%$ confidence interval 0.99 to 7.20 ) was in favour of the screening intervention increasing referrals to supportive services in hospital or community, the absolute numbers of referrals were low and the difference was not significant at the 5\% level.

Data related to information giving, as well as the take up of services, were too heterogeneous to allow meta-analysis of either outcome. For example, Rhodes and colleagues defined provision of services for intimate partner violence as safety assessment, counselling by the health provider/social worker, and/or provision of information about resources. ${ }^{27}$ In that study, $7.6 \%$ $(21 / 262)$ of the screened urban women received services compared with $3.6 \%$ (10/275) of the unscreened women. Among screened suburban women, $2.5 \%$ (4/159) received services compared with none of the 171 unscreened women. Trautman and colleagues found that $4.3 \%$ (18/411) of screened women received social work assistance for intimate partner violence compared with $1 \%(2 / 194)$ of women in the comparison group. ${ }^{21}$ In the study by Klevens and colleagues, 4/36 women (11\%) 
who had face to face screening reported at one week that they had taken up services from the computer printout of resources compared with 2/66 women (3\%) in the computer (comparison) group. ${ }^{24}$ Koziol-McLain and colleagues found no differences on resource use based on the community resource checklist ${ }^{28}$ after three months. ${ }^{23}$ Ahmad and colleagues reported that clinicians discussed safety with nine of the $25(36 \%)$ women identified in the screened group and with only one of $12(8 \%)$ women identified in the comparison group. ${ }^{25}$ Follow-up appointments were set up with 20/25 women in the screened group compared with 8/12 of non-screened women.

\section{Projected benefit of screening in terms of identification and referral}

From a base of 31.8/1000 abused women being identified in usual care, a screening programme would add an additional 42 women (95\% confidence interval 12 to 92). From 7.4/1000 referred in usual care, screening for intimate partner violence would add an additional 12 referrals (7 to 50) (table $2 \Downarrow$ ).

\section{Subgroup analyses}

In subgroup analyses (fig $4 \Downarrow$ ), two studies tested screening in antenatal settings. We estimated a greater than $300 \%$ likelihood of increased identification by clinicians in screened pregnant populations, although we are cautious about this inference as the confidence intervals were wide. In the three studies that evaluated identification from screening in emergency settings, we estimated a $161 \%$ greater likelihood of clinicians identifying women experiencing intimate partner violence. While the effect size was larger in antenatal settings, the confidence intervals overlapped for the effects in the two settings.

\section{Intimate partner violence}

Two studies measured subsequent exposure to intimate partner violence among screened versus non-screened women. Both studies used the composite abuse scale ${ }^{29}$ but had different denominators and timelines. Koziol-McLain and colleagues measured recurrent intimate partner violence after three months among all women. ${ }^{23}$ MacMillan and colleagues measured recurrent intimate partner violence after 6,12 , and 18 months in women who screened positive at baseline. ${ }^{26}$ Both studies found a non-significant reduction in such violence after screening, with similar point estimates of effect: odds ratio 0.86 (95\% confidence interval 0.39 to 1.92 ) in the study by Koziol-McLain and colleagues at three months' follow-up ${ }^{23}$ and 0.88 ( 0.43 to 1.82$)$ at 18 months in the study by MacMillan and colleagues. $^{26}$

\section{Women's physical and psychosocial health}

Only the study by MacMillan and colleagues ${ }^{26}$ measured physical health (SF-12).$^{30}$ At 6,12 , and 18 months, the effect favoured those who received screening, with increases at each time point, but at 18 months, this was no longer a significant effect (mean difference $1.57,95 \%$ confidence interval -0.59 to 3.73). This study was also the only one to measure psychosocial health. Overall, there was no evidence of impact on quality of life or post-traumatic stress disorder, depression, or alcohol and drug problems.

\section{Occurrence of adverse outcomes}

Studies measured women's preferences for screening method, ${ }^{19}$ acceptability, ${ }^{23}{ }^{25}$ comfort levels, ${ }^{22}$ positive and negative reactions, ${ }^{24}$ and overall satisfaction. ${ }^{27}$ Negative reactions to screening were reported as negligible in the trial by Klevens and colleagues. ${ }^{24}$ Koziol-McLain and colleagues reported no adverse effects. ${ }^{23}$ Rhodes and colleagues found that inquiries about and disclosures of intimate partner violence were associated with higher patient satisfaction with care. ${ }^{27}$ Only one study, however, measured potential harm, using a new measure developed for the trial, the consequences of screening tool (COST) ${ }^{26}$ The mean score of 3.52 (SD 3.24) on the eight item effects on quality of life subscale supported the view that being asked screening questions about intimate partner violence was not harmful to women in the short term. No trial included an economic analysis.

\section{Discussion Principal findings}

In this review of the 11 trials testing screening for intimate partner violence versus routine care, we found moderate evidence that screening in healthcare settings in high income countries increases rates of identification of women subject to this sort of violence compared with usual care. This was particularly the case in the two studies set in antenatal settings; in a hospital outpatient setting there was no significant increase in identification. When there was an increase in identification, it was modest compared with the prevalence of intimate partner violence among women attending healthcare settings. ${ }^{2} \mathrm{We}$ found little evidence that screening increases referrals to support services. Furthermore, though not meta-analysed, the trials did not find an impact of screening on improved outcomes for women. Only two studies measured the impact of screening on re-exposure to intimate partner violence and did not find that screening alone reduces abuse. Only one study measured potential harm from screening.

Thus, weighing up the limited evidence of benefit beyond identification and the fact that most studies do not measure the risks of screening, the current evidence does not support screening programmes for intimate partner violence in healthcare settings. There remains an urgent need to conduct trials that will be able to clearly evaluate the potential for such screening to improve women's health and social outcomes and its potential to do harm.

\section{Types and context of studies}

Screening for intimate partner violence in healthcare settings is a complex intervention, which makes the choice of inclusion criteria for a systematic review problematic. Definitions of screening for intimate partner violence are often vague or absent. ${ }^{31}$ We included only studies of interventions that involved a consultation between patient and clinician; we excluded studies of screening programmes that were followed by structured interventions such as advocacy or therapeutic interventions. Studies were conducted in urban and rural settings in predominantly high income countries with legislation and specialist support services for intimate partner violence to which clinicians could refer.

They were situated in a range of healthcare settings. Screening was most effective in the antenatal setting, possibly because screening can offer multiple opportunities for women at a key turning point in their lives. Emergency care settings are subject to high staff turnover, limited ability to sustain training, and a crisis atmosphere, so that the opportunity to help might be limited, but again social work help might be available. Different opportunities exist in primary care, depending on community resources. While more women are identified in antenatal settings, the lack of evidence for improved outcomes suggests 
targeted screening programmes require redesign and possibly more resources (for example, system support and effective sustainable health professional training) followed by trial evaluation to see if there is long term benefit for women and babies. Studies used various screening tools and either deployed clinician screening or communication to the clinician of a positive screening result derived from a self completion method (computer or written survey), compared with usual care. Identification, referral, and distribution of information about intimate partner violence and resources were primary outcomes, although were not used in inclusion criteria. Follow-up time varied from immediately after the intervention for identification rates and referral to 18 months for health outcomes, recurrence of intimate partner violence, and resource use. We acknowledge the fundamental importance of reduction in intimate partner violence as an outcome of these trials. As women have limited control over the violence they experience, however, process outcomes (such as identification and referral) might be more meaningful in studies with short follow-up periods as tends to be the case in screening trials.

\section{Strengths and limitations}

Meta-analysis was limited by heterogeneity in outcomes and approaches to measurement across included studies. In our review, "identification" referred to any form of acknowledgment by a clinician during a consultation that the woman had experienced exposure to intimate partner violence. The clinical interaction was seen as important in increasing the likelihood that a woman with a positive result on screening would receive an appropriate response. Identification might have been underestimated by relying on clinicians' willingness to document intimate partner violence in women's charts ${ }^{21-32}$ or participants' recall of what occurred during encounters. ${ }^{20}{ }^{33}$ Audio-recording encounters to collect data on identification could also have influenced clinicians and patients' willingness to raise or discuss the issue ${ }^{25}{ }^{27}$ Rates of identification by clinicians were low relative to the rates of disclosure in the computerised or written assessment. For example, Rhodes and colleagues found that of the 83 participants in the intervention group who reported intimate partner violence on the computer self assessment (that was subsequently conveyed to the clinician), just 19 cases were documented by clinicians in patients' charts. In another study, just $48 \%$ of encounters with a prompt for clinicians led to a discussion of intimate partner violence. ${ }^{27}$ This reflects the barriers that both women and clinicians experience in relation to approaching the problem of intimate partner violence in women's lives. ${ }^{10}{ }^{34}$ It was also difficult to use referral as an outcome for analysis, with some studies defining it as the provision of lists of resources/services and others alluding to more formal processes of immediate referral to social work and other clinical/support services. Thus, future trials need to be attuned to the methodological challenge of operationalising outcomes in this context,,$^{35}$ including not treating the provision of a list of services as "a referral." One of the criticisms commonly raised against the implementation of screening is that there has been insufficient study of whether screening for intimate partner violence in healthcare settings can be harmful to women. ${ }^{36}$ The one trial that systematically assessed harm was reassuring on that count. ${ }^{26} \mathrm{~A}$ recent trial that added a brief counselling intervention after screening and used the same harm measure also reported no adverse effect of screening. ${ }^{37}$ Monitoring harm with specific validated measures is an imperative for future studies dealing with intimate partner violence in healthcare settings. None of the trials included an economic analysis. Both the proportions of women choosing not to disclose and the impact of false identification on women's lives need further investigation before we can fully understand the effectiveness of screening.

Only four studies were assessed as being at low risk of bias for four of the seven domains examined. While nine studies minimised the risk of selection bias through effective random sequence allocation, lack of blinding of participants and providers (performance bias), and the associated potential for differential behaviours across study groups, was the most consistent threat to validity generally. This was unsurprising given that it is difficult if not impossible to blind providers in studies of complex interventions. This is particularly true in a cluster randomised design such as a study that sought to determine if clinicians using a particular assessment form detected more antenatal psychosocial concerns in pregnant women compared with clinicians providing usual prenatal care. ${ }^{20}$ Despite the difficulties in minimising performance bias, cluster trials do protect against contamination associated with crossover effects. Another limitation arises from the complex ethical challenges of conducting true randomised experiments of services for those experiencing intimate partner violence: most studies involved offering some relevant information to participants in the comparison arm. Thus, future trials need to weigh up the costs and benefits of minimising the risk of bias because, while reducing bias will optimise the validity of study findings, it must also be feasible and ethical. Studies in intimate partner violence can be particularly susceptible to contextual factors because of the sensitive and stigmatised nature of such violence. This underscores the need for qualitative and process modelling methods to investigate external factors impacting on outcomes. ${ }^{38}$ Few studies reported a process evaluation, and the published reports often lacked detail about organisational context and how clinicians were trained and supported to undertake screening.

\section{Implications for clinicians and policymakers}

Although some governments and healthcare policy and professional associations mandate screening for intimate partner violence,,${ }^{40}$ that position is not consistent with previous systematic reviews ${ }^{5-41}$ and conflicts with the recent guidance from the UK National Institute for Health and Care Excellence $(\mathrm{NICE})^{42}$ and $\mathrm{WHO} \cdot{ }^{43}$ Neither this systematic review nor the totality of evidence from other reviews support the implementation of routine screening for intimate partner violence at this time. Although identification of affected women patients increases, the absolute number of women identified is modest in comparison with the numbers of women screened and the likely prevalence of intimate partner violence, so a screening programme might provide false reassurance to healthcare providers and policymakers. Moreover, from the small number of screening trials that measured health outcomes for women, there is insufficient evidence of benefit derived to the women screened.

\section{Unanswered questions and future research}

Future trials should be conducted to provide evidence for whether violence decreases and whether women's wellbeing increases. To do this, trials must be carried out in a setting with adequate resources to support the women identified. It is essential that future research recognises the complexity of trial design, implementation, and evaluation. There is a need for well defined theoretically informed interventions, a rationale for outcome selection and measurement, and investigation of context and process. Finding innovative approaches to minimising performance bias as well as increased description (in process 
evaluation and published protocols) could strengthen the evidence coming from future studies. Cluster randomisation without recruitment of individual participants is one ethical method for designing trials of interventions for intimate partner violence, with patients unaware that they are in a trial. ${ }^{44}$ More studies are needed that examine different methods of identification of affected women, including a range of healthcare settings and diverse populations. We must examine the social and economic benefits for the differing strategies of screening versus case finding. The question of whether to move towards targeting screening to subgroups of high risk women also remains unanswered. A criticism of screening trials generally is insufficient intervention after disclosure. More research is necessary to evaluate the effectiveness of screening linked with advocacy, ${ }^{45}$ social support, ${ }^{46}$ and structured clinician support ${ }^{37}$ and other therapeutic interventions. Generally, we need to shift research effort towards testing the effectiveness of interventions for women who disclose abuse, whatever the method of identification. ${ }^{47}$

We thank the Australasian Cochrane Centre for training and support; the Cochrane Developmental, Psychosocial and Learning Problems Group for their support and assistance with searches and publication; and Cochrane Collaboration for funding support (training).

This article is based on a Cochrane Review ${ }^{12}$ (see www.

thecochranelibrary.com for information). Cochrane Reviews are regularly updated as new evidence emerges and in response to feedback, and the Cochrane Database of Sytematic Reviews should be consulted for the most recent version of the review.

Contributors: The original search strategy was developed and executed by AT in collaboration with Liesje Toomey, Jo Abbott, and Margaret Anderson of the Cochrane Developmental, Psychosocial and Learning Problems Group. AT, LO'D, and KH selected studies; LO'D and AT extracted the data; AT undertook the analysis with the help of LO'D and $\mathrm{KH}$, and drafted the original Cochrane review. All authors provided topic expertise and contributed to writing and editing the review. LO'D and AT drafted the abridged version with editing input from all authors. LO'D and AT are guarantors.

Funding: La Trobe University provided funding for work undertaken towards the original Cochrane Review; the university had no role in the research or its publication.

Competing interests: All authors have completed the ICMJE uniform disclosure form at www.icmje.org/coi_disclosure.pdf and declare: no support from any organisation for the submitted work; no financial relationships with any organisations that might have an interest in the submitted work in the previous three years; Kelsey Hegarty and Gene Feder participated in the WHO guideline group on health practitioners' response to intimate partner violence; Gene Feder chaired the programme development group of the UK NICE domestic violence and abuse guidelines; no other relationships or activities that could appear to have influenced the submitted work.

Ethical approval: Not required.

Data sharing: Further data are available from a.taft@latrobe.edu.au or original Cochrane review at http://onlinelibrary.wiley.com/doi/10.1002/ 14651858.CD007007.pub2/abstract.

Transparency: The lead author (the manuscript's guarantor) affirms that the manuscript is an honest, accurate, and transparent account of the systematic review being reported; that no important aspects of the review have been omitted; and that any discrepancies from the study as planned (and, if relevant, registered) have been explained.

1 Ellsberg M, Jansen HA, Heise L, Watts $\mathrm{CH}$, Garcia-Moreno C. Intimate partner violence and women's physical and mental health in the WHO multi-country study on women's health and domestic violence: an observational study. Lancet 2008;371:1165-72
2 World Health Organization. Global and regional estimates of violence against women: prevalence and health effects of intimate partner violence and non-partner sexual violence. WHO, 2013.

3 Krug EG, Mercy JA, Dahlberg LL, Zwi AB. The world report on violence and health. Lancet 2002;360:1083-8

4 Davidson LL, King V, Garcia J, Marchant S. Reducing domestic violence... what works? Health Services. Home Office, 2000.

5 World Health Organization. Responding to intimate partner violence and sexual violence against women: WHO clinical and policy guidelines. WHO, 2013.

6 Moyer VA. Screening for intimate partner violence and abuse of elderly and vulnerable adults: US preventive services task force recommendation statement. Ann Intern Med 2013;158:478-86

7 Bacchus L, Bewley S, Fernandez C, Hellbernd H, Lo Fo Wong S, Otasevic S, et al. Health sector responses to domestic violence in Europe: a comparison of promising intervention models in maternity and primary care settings. London School of Hygiene and Tropical Medicine, 2012

8 Feder G, Ramsay J, Dunne D, Rose M, Arsene C, Norman R, et al. How far does screening women for domestic (partner) violence in different health-care settings meet criteria for a screening programme? Systematic reviews of nine UK National Screening Committee criteria. Health Technol Assess 2009:13:iii-iv, xi-xiii, 1-113, 137-347.

9 Nelson HD, Bougatsos C, Blazina I. Screening women for intimate partner violence: a systematic review to update the 2004 Preventive Services Task Force recommendation. Ann Intern Med 2012;156:1-13;W11-3.

10 Hegarty KL, Taft AJ. Overcoming the barriers to disclosure and inquiry of partner abuse for women attending general practice. Aust N Z J Public Health 2001;25:433-7.

11 Hegarty K, Feder G, Ramsay J. Identification of partner abuse in health care settings: should health professionals be screening? In: Roberts G, Hegarty, K, Feder, G, ed. Intimate partner abuse and health professionals. Elsevier, 2006.

12 Taft A, O'Doherty L, Hegarty K, Ramsay J, Davidson L, Feder G. Screening women for intimate partner violence in healthcare settings. Cochrane Database Syst Rev 2013;4:CD007007.

13 Devine A, Spencer A, Eldridge S, Norman R, Feder G. Cost-effectiveness of Identification and Referral to Improve Safety (IRIS), a domestic violence training and support programme for primary care: a modelling study based on a randomised controlled trial. BMJ Open 2012;2.

14 Feder G, Davies R, Baird K, Dunne D, Eldridge S, Griffiths C, et al. Identification and Referral to Improve Safety (IRIS) of women experiencing domestic violence with a primary care training and support programme: a cluster randomised controlled trial. Lancet 2011:378:1788-95

15 Taft A, Hegarty K, Ramsay J, Feder G, Carter Y, Davidson L, et al. Screening women for intimate partner violence in health care settings (protocol). Cochrane Database Syst Rev 2009;1:CD007007.

16 Cochrane Collaboration. Review Manager (RevMan). Nordic Cochrane Centre, 2011.

17 Higgins JPT, Green S, eds. Cochrane handbook for systematic reviews of interventions. Version 5.1.0 (updated March 2011). Cochrane Collaboration, 2011.

18 GRADE Working Group. Grading quality of evidence and strength of recommendations. BMJ 2004;328:1490.

19 MacMillan HL, Wathen CN, Jamieson E, Boyle M, McNutt LA, Worster A, et al. Approaches to screening for intimate partner violence in health care settings: a randomized trial. JAMA 2006;296:530-6.

20 Carroll JC, Reid AJ, Biringer A, Midmer D, Glazier RH, Wilson L, et al. Effectiveness of the Antenatal Psychosocial Health Assessment (ALPHA) form in detecting psychosocial concerns: a randomized controlled trial. CMAJ 2005;173:253-59.

21 Trautman DE, McCarthy ML, Miller N, Campbell JC, Kelen GD. Intimate partner violence and emergency department screening: computerized screening versus usual care. Ann Emerg Med 2007;49:526-34.

22 Kataoka $\mathrm{Y}, \mathrm{Yaju} \mathrm{Y}, \mathrm{E}$ to $\mathrm{H}$, Horiuchi S. Self-administered questionnaire versus interview as a screening method for intimate partner violence in the prenatal setting in Japan: a randomised controlled trial. BMC Pregnancy Childbirth 2011;10:84.

23 Koziol-McLain J, Garrett N, Fanslow J, Hassall I, Dobbs T, Henare-Toka TA, et al. A randomized controlled trial of a brief emergency department intimate partner violence screening intervention. Ann Emerg Med 2010;56:413-23.

24 Klevens J, Sadowski L, Kee R, Trick W, Garcia D. Comparison of screening and referral strategies for exposure to partner violence. Womens Health Issues 2012;22:e45-52.

25 Ahmad F, Hogg-Johnson S, Stewart DE, Skinner HA, Glazier RH, Levinson W. Computer-assisted screening for intimate partner violence and control: a randomized trial. Ann Intern Med 2009;151:93-102.

26 MacMillan HL, Wathen CN, Jamieson E, Boyle MH, Shannon HS, Ford-Gilboe M, et al. Screening for intimate partner violence in health care settings: a randomized trial. JAMA 2009:302:493-501.

27 Rhodes KV, Drum M, Anliker E, Frankel RM, Howes DS, Levinson W. Lowering the threshold for discussions of domestic violence: a randomized controlled trial of computer screening. Arch Intern Med 2006;166:1107-14.

28 McFarlane J, Malecha A, Gist J, Watson K, Batten E, Hall I, et al. Increasing the safety-promoting behaviors of abused women. Am J Nurs 2004:104:40-50

29 Hegarty K, Bush R, Sheehan M. The composite abuse scale: further development and assessment of reliability and validity of a multidimensional partner abuse measure in clinical settings. Violence Vict 2005;20:529-47.

30 Ware JJ, Kosinski M, Keller SD. A 12-item short-form health survey: construction of scales and preliminary tests of reliability and validity. Med Care 1996;34:220-33.

31 Wathen CN, MacGregor JC, Sibbald SL, MacMillan HL. Exploring the uptake and framing of research evidence on universal screening for intimate partner violence against women: a knowledge translation case study. Health Res Policy Syst 2013;11:13.

32 Rhodes KV, Lauderdale DS, He T, Howes DS, Levinson W. "Between me and the computer": increased detection of intimate partner violence using a computer questionnaire. Ann Emerg Med 2002;40:476-84.

33 Humphreys J, Tsoh JY, Kohn MA, Gerbert B. Increasing discussions of intimate partne violence in prenatal care using Video Doctor plus Provider Cueing: a randomized, controlled trial. Womens Health Issues 2011;21:136-44.

34 Colombini M, Mayhew S, Watts $\mathrm{C}$. Health-sector responses to intimate partner violence in low- and middle-income settings: a review of current models, challenges and opportunities. Bull World Health Organ 2008;86:635-42.

35 Taft A, Hegarty K. Intimate partner violence against women: what outcomes are meaningful? JAMA 2010;304:577-9.

36 Jewkes R. Preventing domestic violence. BMJ 2002;324:253-4. 


\section{What is already known on this topic}

WHO highlights violence against women as a priority health issue, with one in three women globally experiencing physical and/or sexual violence from a partner

Despite the lack of evidence of impact of screening programmes for intimate partner violence on women's health and social outcomes, several professional associations internationally advocate screening all women in health settings, and it is national policy in some countries

\section{What this study adds}

Despite an increase in identification from screening, this was low relative to the prevalence of intimate partner violence; it was unclear whether screening increases effective referral; and the review detected neither improved outcomes for women nor harm from screening

There is insufficient evidence for universal screening in healthcare settings. Studies comparing screening versus case finding or screening in combination with therapeutic intervention for women's long term wellbeing are needed to inform the implementation of identification policies for intimate partner violence in healthcare settings

37 Hegarty K, O'Doherty L, Taft A, Chondros P, Brown S, Valpied J, et al. Screening and counselling in the primary care setting for women who have experienced intimate partner violence (WEAVE): a cluster randomised controlled trial. Lancet 2013;382:249-58.

38 Spangaro J, Zwi AB, Poulos R. The elusive search for definitive evidence on routin screening for intimate partner violence. Trauma Violence Abuse 2009;10:55-68.

39 May CR, Finch T, Ballini L, MacFarlane A, Mair F, Murray E, et al. Evaluating complex interventions and health technologies using normalization process theory: development of a simplified approach and web-enabled toolkit. BMC Health Serv Res 2011:11:245.

40 Family Violence Prevention Fund. National consensus guidelines on identifying and responding to domestic violence victimisation in health care settings. Family Violence Prevention Fund, 2004.

41 Hegarty K. What is intimate partner abuse and how common is it? In: Roberts G, Hegarty $\mathrm{K}$, Feder G, eds. Intimate partner abuse and health professionals: new approaches to domestic violence. Elsevier, 2006:19-40.

42 National Institute for Health and Care Excellence. Domestic violence and abuse: how health services, social care and the organisations they work with can respond effectively. NICE, 2014.

43 Feder G, Wathen CN, MacMillan HL. An evidence-based response to intimate partner violence: WHO guidelines. JAMA 2013;310:479-80.

44 Eldridge SM, Ashby D, Feder GS. Informed patient consent to participation in cluster randomized trials: an empirical exploration of trials in primary care. Clinical Trials 2005;2:91-8.
45 Ramsay J, Carter Y, Davidson L, Dunne D, Eldridge S, Feder G, et al. Advocacy interventions to reduce or eliminate violence and promote the physical and psychosocial well-being of women who experience intimate partner abuse. Cochrane Database Syst Rev 2009;3:CD005043.

46 Taft AJ, Small R, Hegarty KL, Watson LF, Gold L, Lumley J. Mothers' AdvocateS In the Community (MOSAIC) -non-professional mentor support to reduce intimate partner violence and depression in mothers: a cluster randomised trial in primary care. $B M C$ Public Health 2011:11:1-10.

47 Wathen $\mathrm{CN}$, MacMillan HL. Health care's response to women exposed to partner violence: moving beyond universal screening. JAMA 2012;308:712-3.

\section{Accepted: 10 April 2014}

\section{Cite this as: BMJ 2014;348:g2913}

This is an Open Access article distributed in accordance with the Creative Commons Attribution Non Commercial (CC BY-NC 3.0) license, which permits others to distribute, remix, adapt, build upon this work non-commercially, and license their derivative works on different terms, provided the original work is properly cited and the use is non-commercial. See: http://creativecommons.org/licenses/by-nc/3.0/. 


\section{Tables}

Table 1| Risk of bias summary: review authors' judgments about each risk of bias item for each included study of screening women for intimate partner violence in healthcare settings (adapted from Taft et $\mathrm{al}^{12}$ )

\begin{tabular}{|c|c|c|c|c|c|c|c|}
\hline & $\begin{array}{l}\text { Random sequence } \\
\text { generation } \\
\text { (selection bias) }\end{array}$ & $\begin{array}{c}\text { Allocation } \\
\text { concealment } \\
\text { (selection bias) }\end{array}$ & $\begin{array}{c}\text { Blinding of } \\
\text { participants and } \\
\text { personnel } \\
\text { (performance bias) }\end{array}$ & $\begin{array}{l}\text { Blinding of } \\
\text { outcome } \\
\text { assessment } \\
\text { (detection bias) }\end{array}$ & $\begin{array}{c}\text { Incomplete } \\
\text { outcome data } \\
\text { (attrition bias) }\end{array}$ & $\begin{array}{c}\text { Selective } \\
\text { reporting } \\
\text { (reporting bias) }\end{array}$ & Other bias \\
\hline Ahmad, 2009 & Low & Low & High & Low & High & Unclear & High \\
\hline Carroll, 2005 & Low & Unclear & High & High & High & High & Unclear \\
\hline Humphreys, 2011 & Low & Low & High & High & Unclear & Unclear & High \\
\hline Kataoka, 2010 & Low & Unclear & Unclear & Low & Low & Low & High \\
\hline Klevens, 2012a & Low & Low & Low & High & Low & Unclear & High \\
\hline Koziol-McLain, 2010 & Low & Low & High & Low & Unclear & Unclear & Low \\
\hline MacMillan, 2006 & Low & High & High & High & Unclear & Low & High \\
\hline MacMillan, 2009 & Low & High & High & Low & Low & Low & High \\
\hline Rhodes, 2002 & High & Unclear & High & Low & Unclear & Low & High \\
\hline Rhodes, 2006 & Low & Low & High & Unclear & Unclear & High & High \\
\hline Trautman, 2007 & High & High & High & Unclear & Unclear & Unclear & High \\
\hline
\end{tabular}


Table 2/ Screening for intimate partner violence compared with no screening or screening for another purpose

\begin{tabular}{|c|c|c|c|c|c|c|}
\hline \multirow[b]{2}{*}{ Outcomes } & \multicolumn{3}{|c|}{ Illustrative comparative benefit* $(95 \% \mathrm{Cl})$} & \multirow[b]{2}{*}{$\begin{array}{l}\text { Relative effect‡ } \\
\quad(95 \% \mathrm{Cl})\end{array}$} & \multirow{2}{*}{$\begin{array}{c}\text { No of } \\
\text { participants } \\
\text { (studies) }\end{array}$} & \multirow[b]{2}{*}{$\begin{array}{l}\text { Quality of } \\
\text { evidence§ }\end{array}$} \\
\hline & $\begin{array}{l}\text { Assumed benefit: } \\
\text { unscreened per } 1000\end{array}$ & $\begin{array}{l}\text { Corresponding benefit: } \\
\text { screened per } 1000\end{array}$ & $\begin{array}{c}\text { Absolute benefit per } \\
1000 \dagger\end{array}$ & & & \\
\hline Identification & 32 & $74(44$ to 124$)$ & 42 (12 to 92$)$ & 2.33 (1.39 to 3.89$)$ & $3564(6)$ & Moderate \\
\hline Referral & 7 & $19(7$ to 50$)$ & $12(0$ to 43$)$ & 2.67 (0.99 to 7.20$)$ & $1400(3)$ & Low \\
\hline
\end{tabular}

*Assumed benefit was from sum of identified or referred but unscreened women and calculated for rate per 1000. Corresponding benefit and confidence intervals calculated by multiplying assumed benefit by risk ratio $(R R)$ and $\mathrm{Cls}$ of unscreened to screened women.

†Absolute benefit calculated with formula $1000 \times A C R \times(1-R R)$ where ACR is assumed control risk and based on number per $1000 .^{17}$

‡Risk ratio.

§GRADE Working Group grades of evidence. ${ }^{18}$ 


\section{Figures}

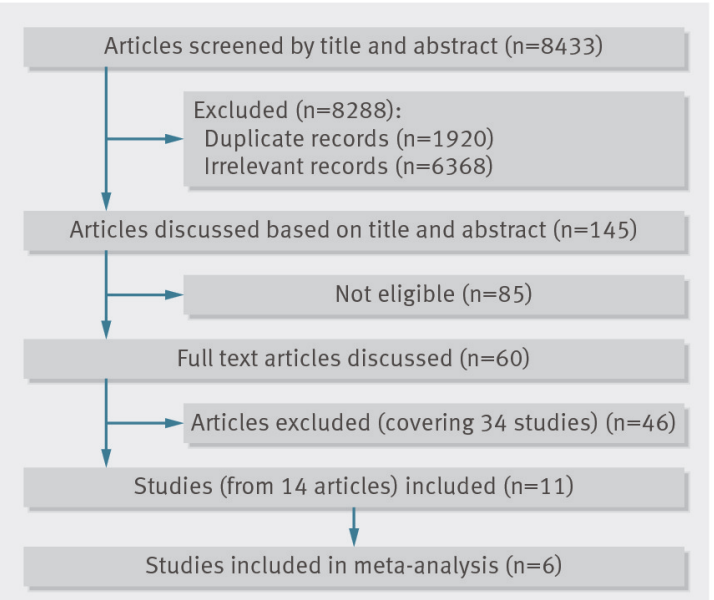

Fig 1 Flow diagram for selection of studies of screening for intimate partner violence in healthcare settings

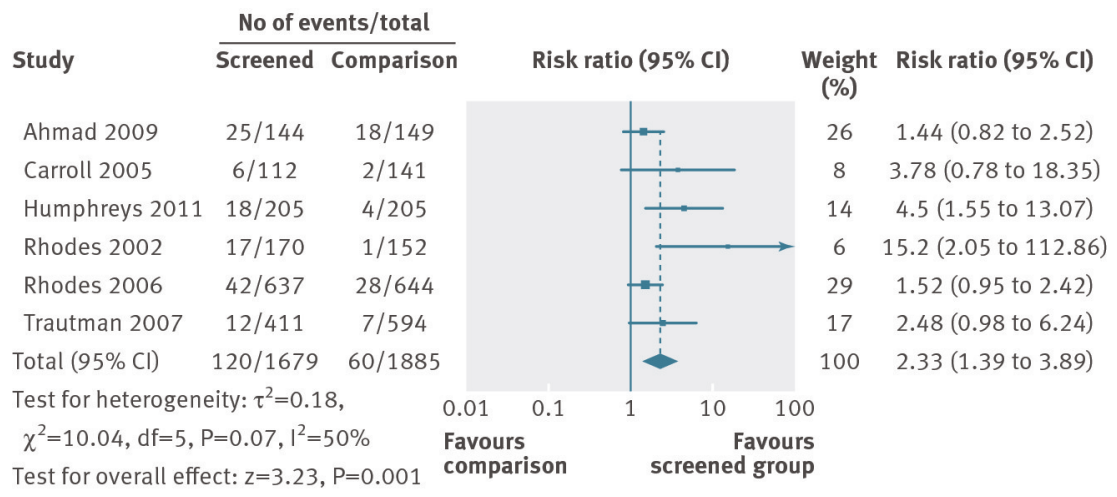

Fig 2 Effect of screening $v$ usual care on identification of intimate partner violence in healthcare settings

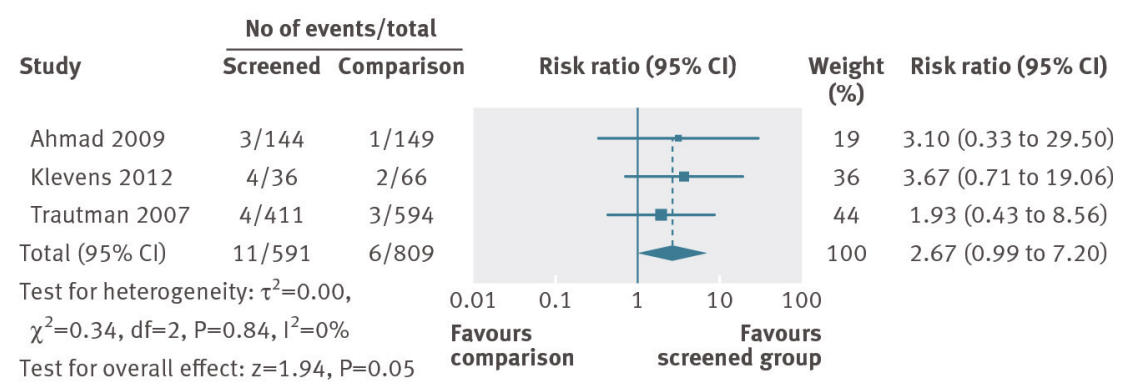

Fig 3 Effect of screening versus comparison on referrals for intimate partner violence in healthcare settings 


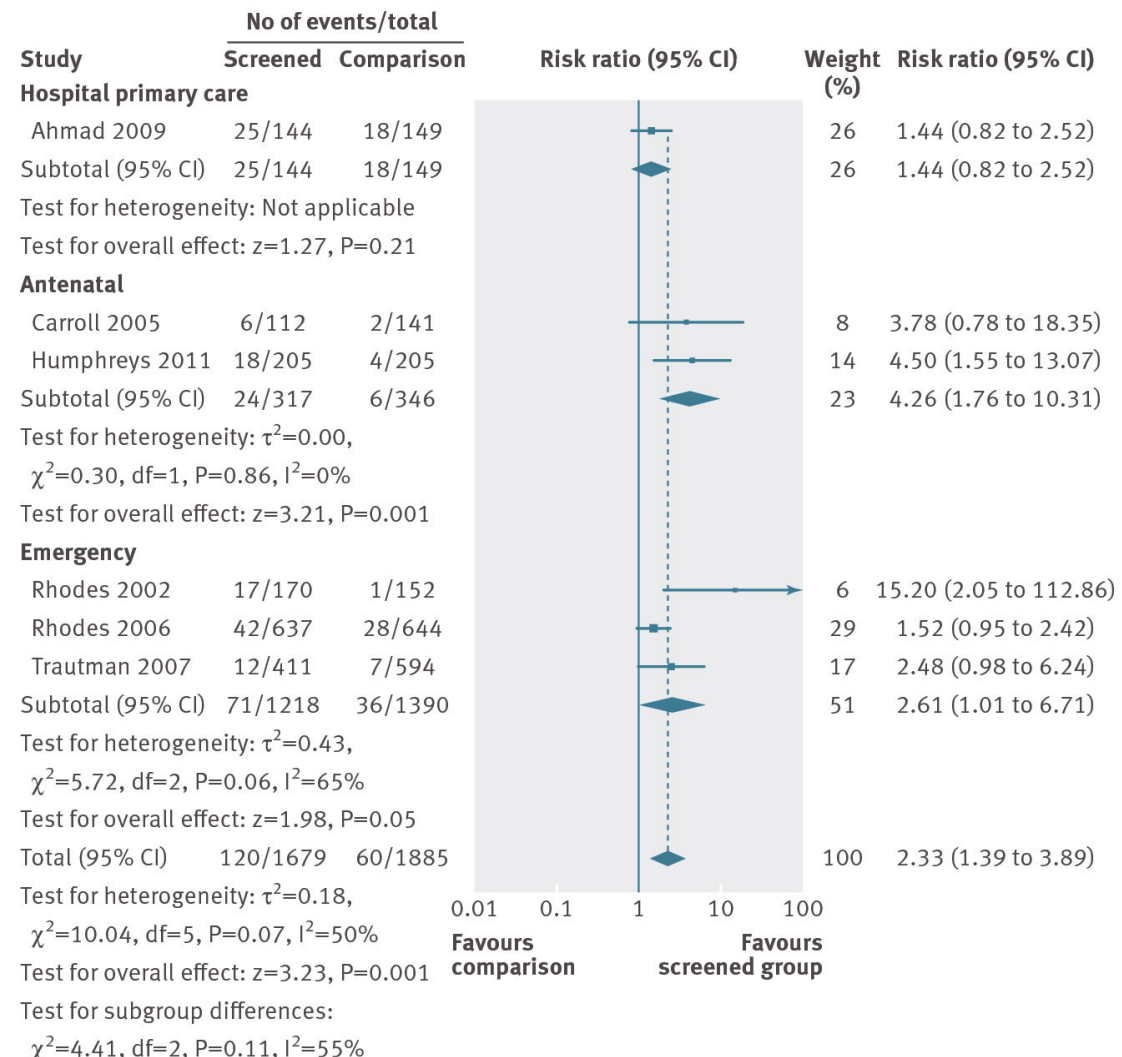

$11,\left.\right|^{2}=55 \%$

Fig 4 Effect of screening on identification of women experiencing intimate partner violence by location subgroup 